\title{
CFD Simulation of Flow around External Vehicle: Ahmed Body
}

\author{
Saurabh Banga ${ }^{1}$, Md. Zunaid ${ }^{2 *}$, Naushad Ahmad Ansari ${ }^{3}$, Sagar Sharma ${ }^{4}$, \\ Rohit Singh Dungriyal ${ }^{5}$ \\ Assistant Professor, Department of Mechanical Engineering, Delhi Technological University, Bawana Road, \\ Delhi, India ${ }^{2,3}$ \\ U.G Student, Department of Mechanical \& Automobile Engineering, Delhi Technological University, Bawana
} Road, Delhi, India ${ }^{1,4,5}$

\begin{abstract}
The main objective of this paper is to elucidate a solution to reduce the inefficiencies and losses caused due to major parameters pertaining to the aerodynamics of road vehicle (drag, lift). This is accomplished by investigating the variation of the rear slant angle of the Ahmed body and its effect on the drag and lift coefficients and determine the optimum angle for least drag through numerical simulation. The simplified vehicle geometry: Ahmed Reference Model has been used as a benchmark. The computational part consists of numerical simulation of the flow around the Ahmedbody employing CFD (Computational Fluid Dynamics) techniques. In numerical simulation results, pressure-based solver was utilized and the turbulence model employed was $k$-Epsilon realizable model with non-equilibrium wall function for near wall treatment. The designing of model used in this paper is done through the SOLIDWORKS '14; and CFD simulation carried out in FLUENT (ANSYS 14).
\end{abstract}

Keywords: Ahmed body, CFD, $k$-Epsilon realizable model, Drag, Lift

\section{Introduction}

The pollution emerged by using volatile fuels in automobile has become a major concern pertaining to the environment and economic viability. Different approaches such as improving the efficiency or usage of alternative fuels for the vehicle have been considered. Advancement in some of the major areas such as aerodynamics improvement, weight reduction and power increase has given us many feasible results, but reducing aerodynamic factors such as drag, lift is more efficient than increasing the power of the vehicle or decreasing the weight of the vehicle.

Automotive aerodynamics comprises of the study of aerodynamics of road vehicles. Its main goals are reducing drag, minimizing noise emission, improving fuel economy, preventing undesired lift forces and minimising other causes of aerodynamic instability at high speeds. Also, in order to maintain better control for steering and braking, we look into design and aerodynamics of a vehicle. Drag is caused due to the pressure difference between the frontal and the rear end of the vehicle. It can be reduced by modification of the design of the vehicle or the modification of the air flow around the vehicle. 50\% of the mechanical energy of the vehicle is wasted to overcome drag at highway speed of nearly 88.5 to $96.5 \mathrm{kph}{ }^{[1]}$

It is necessary, at times, to generate down force - to improve traction and thus cornering abilities.Lift can be dangerous for an automobile, especially at high speeds. So, in order to maintain control for steering and braking, cars are designed so that the automobile exerts a downward force as their speed increases. However, increasing this downward force increases drag, which in turn, limits the top speed and increases fuel consumption. Hence, these two forces must be carefully balanced.

Air has a tendency to curl downwards around the ends of a car, travelling upwards from the highpressure region under the car to the low-pressure region on top, at the rear end of the automobile and subsequently collides with moving low-pressure air. The kinetic energy of these turbulent air spirals acts in a direction that is negative relative to the direction of travel intended. Thus, the car engine must compensate for the losses created by this drag. Vortices are released during flow separation and trail downstream to form structured or unstructured wake patterns. A wake is the region of re-circulating flow immediately behind a moving or stationary solid body, caused by the flow of surrounding fluid around the body. The local disturbances in the flow pattern behind the vehicle causes a momentum loss thus causing form drag which extends far behind the bodyworks of a vehicle.

The generic Ahmed Body reference model has been chosen as the benchmark for carrying out computations for studying of the aerodynamic parameters. The body was first proposed by Ahmed et al., (1984). ${ }^{[2]}$ The Ahmed body is a very simple bluff body which has its shape simple enough to allow for accurate flow simulation but retains some important practical features relevant to automobile bodies. It has a slant on its rear end, whose angle can be manipulated and the corresponding drag and lift coefficients calculated. This is done to exhibit the air flow over the different geometry sections of an automobile and in its vicinity, at different slant 
angles. This model portrays how to calculate the turbulent flow field around a simple car-like geometry using the turbulent flow, k-epsilon interface.

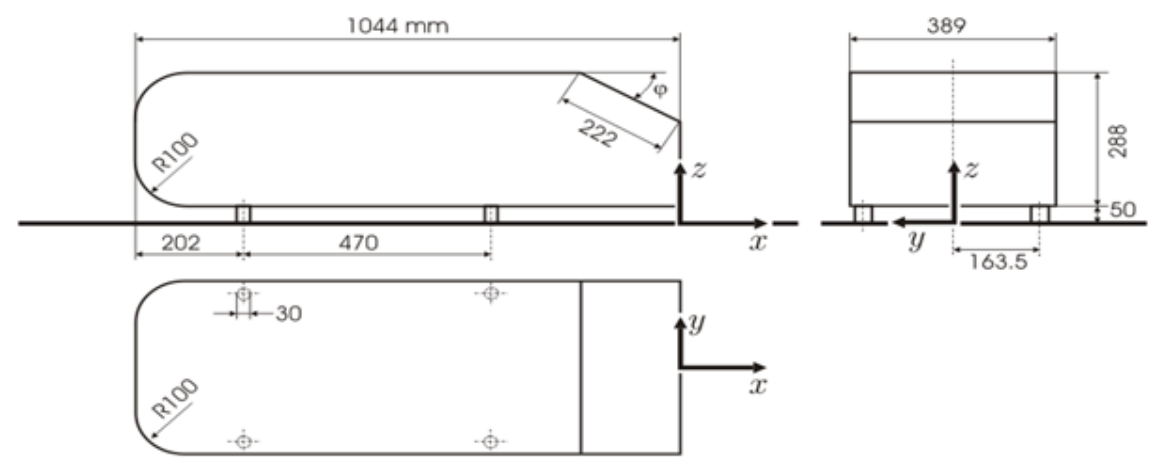

Figure 1: Geometry of Ahmed model

Different rear slant angles result in the emergence of different wake regions at the rear, which are mainly responsible for the drag of the vehicle. This paper has the following objectives:

1. To understand the drag and lift mechanism by conducting analysis on Ahmed body with 10 different angle ranging from 0-40 degrees.

2. To exhibit and comprehend the velocity contours, focussing at the wake region of the body.

3. To portray pressure distribution over the entire Ahmed body.

\section{Literature Review}

Ahmed et al. (1984) ${ }^{[2]}$ analysed time averaged wake structure around the Ahmed body at a Reynolds number equal to $1.2 * 10^{6}$ by manipulating the rear slant angle in the range of $0-40^{0}$ in increments of $5^{0}$. However, the study did not yield information about unsteady flow characteristics of the flow around the Ahmed body.

Bayraktar et al. (2001) ${ }^{[3]}$ examined the external aerodynamics of Ahmed reference body for the rear slant angles of $0,12.5$, and 25 degrees. The main concern was observing the effect on the lift and drag coefficients due to variation of Reynolds number and calculating wind-averaged drag coefficients. The 10-inch water column possessing an electronic scanning module coupled with a force balance system was utilized to measure the pressure and forces which are consequently used to calculate the drag coefficients.

Spohn and Gillieron (2002) ${ }^{[4]}$ carried out experiments to investigate the flow characteristics of the Ahmed body with the slant angle equal to 25 degree and the Reynolds number equal to $8.3 * 10^{3}$. The experiment was carried out in a closed water tunnel, using the electrolyte precipitation technique at the front as well as the rear of the Ahmed body.

M. Zunaid, Naushad Ahmad Ansari ${ }^{[5]}$ designed and implemented the curved boat tail configuration without the AFC device on a generic SUV design working on the principle of Coanda effect, causing the airflow over and under the SUV to take a curved path resulting in blowing of air in the immediate wake region. They examined its effect on various aerodynamic parameters and reported an increase in the average pressure in the wake region along with a significant drag reduction of $8.013 \%$.

Morelli et al. (1976) conceptualized and proposed a theoretical method to determine the optimum shape of a passenger car body for minimum drag by imposing the condition that the total lift be zero. This study proved that the aerodynamic drag can be reduced substantially without utilizing any additional devices by optimizing the body shape. Morelli et al. (2000) ${ }^{[6]}$ proposed a new technique called "fluid tail", obtainable by addressing small power air jets in the wake, for application to the aerodynamic design of basic shape of a passenger car. .

P. Drage et al (2008) ${ }^{[7]}$ conducted both CFD simulations as well as wind tunnel testing (T.U Graz wind tunnel) on the Ahmed body with two rear slant angle configurations. The simulation was performed with the Fluent and by using the Reynold Stress Model. The mesh was developed with the meshing tool SPIDER. It consisted of 4 prism layers around the body and had an unstructured volume mesh giving a total cell count of 7.7 million cells. This type of meshing approach is commonly adopted as the time spent to create a structured grid around real vehicle shapes would prevent engineers from operating within the strict deadlines imposed by vehicle design cycles. For the $25^{\circ}$ angle, a drag coefficient of 0.299 and a lift coefficient of 0.345 was obtained from the wind tunnel experiment, while the CFD simulation resulted in the values of 0.295 and 0.387 for drag and lift coefficient respectively. The RSM model showed an over estimation of the pressure coefficient over the slant angle, when compared to the pressure measurement made by Lienhart and Becker (2003). ${ }^{[8]}$ 
Han (1992) [9] performed aerodynamic shape optimization on Ahmed body with three shape parameters: backlight angle $\left(0^{\circ}\right.$ to $\left.30^{\circ}\right)$, boat tail angle $\left(0^{\circ}\right.$ to $\left.30^{\circ}\right)$ and ramp angle $\left(0^{\circ}\right.$ to $\left.20^{\circ}\right)$. The k- $\varepsilon$ turbulence model CFD solver was coupled with an optimization routine. In this study, an analytic approximation function of the objective function (drag coefficient values from CFD 28 analysis) was created in terms of the design variables. The optimization was then performed on this approximation function and optimum parameters were found. The CFD analysis was again performed with this optimum set of parameters and the objective function was updated with new results. This process was continued until the parameters for minimum drag were obtained. The optimization process revealed that the optimum rear body parameters are backlight angle of $17.8^{0}$; boat-tail angle of $18.9^{0}$; and ramp angle of $9.2^{\circ}$. The determined values for minimum drag were found to lie within the experimentally determined values of $15-18^{0}$ backlight angles, $15-22^{0}$ boat-tail angles and $9-14^{0}$ ramp angles. The drag coefficient was reduced from 0.209 for a square back to 0.110 for an optimized geometry. However, the technique used for parameterization of geometry in this study cannot be applied to complex geometries.

S. Kapadia et. al. ${ }^{[10]}$ conducted CFD analysis on the Ahmed body with the $25^{\circ}$ slant angle configuration using two different turbulence modelling approaches: Detached eddy simulation (DES) and single equation unsteady RANS model. An unstructured volume mesh grid of 1.7 million cells was generated with Gridgen and COBALT was used as a solver. Although both the models predict the presence of the counter rotating vortices, the DES depicted a better representation of the unsteady structure at the trailing edge of the Ahmed body. The drag coefficient reached a constant value after approximately 3 seconds of simulation for both DES and RANS. DES showed an average Cd value of 0.2585 while with the RANS approach the drag the average Cd calculated is 0.3272 .

\section{Numerical Simulation}

The reference Ahmed Body is $1044 \mathrm{~mm}$ long, $327 \mathrm{~mm}$ wide and $288 \mathrm{~mm}$ high. In ANSYS '14 Design Modeller, a single body domain of air is created surrounding the Ahmed body walls after subtracting it from the air enclosure. It has dimensions $5 \mathrm{~m}$ from front, $7.5 \mathrm{~m}$ from the rear and $3 \mathrm{~m}$ from the top.

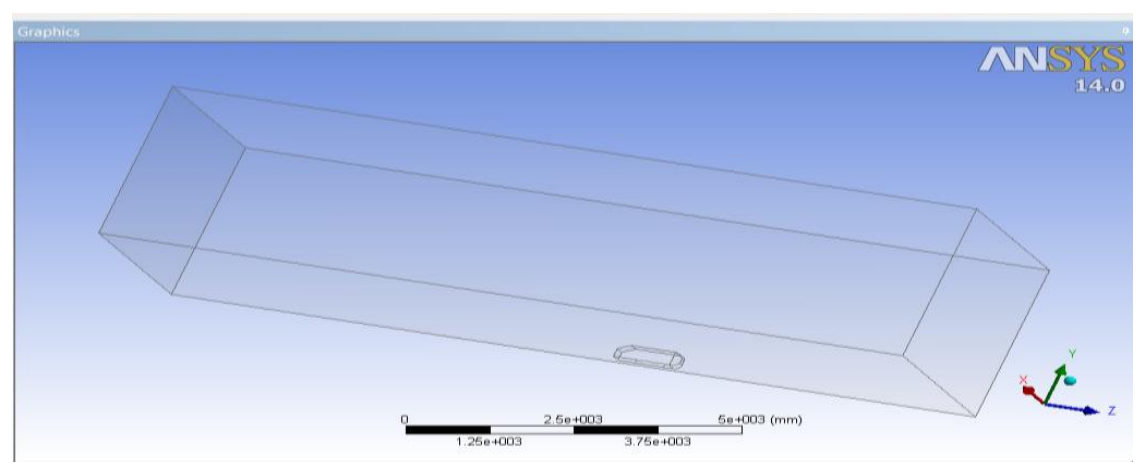

Figure 2: Single air body domain

\subsection{Meshing}

The "finite element method" technique is used in our problem. The ANSYS Meshing Tool is used for carrying out the meshing. The model is $50 \mathrm{~mm}$ above the floor.

Details of the mesh are as follows: Relevance center: coarse, Smoothing: high, Transition: slow, Initial size seed: Active assembly, Min. size: 1mm, Max. Size: 250mm, Advanced Size function: Proximity and Curvature. Modifications in meshing: 
- Size of roller meshing is limited to $2 \mathrm{~mm}$.

- Size of faces meshing is limited to $10 \mathrm{~mm}$.

- Inflation Layer method ${ }^{[11]}$ of first aspect ratio of 5, growth rate of $20 \%$ of 5 layers is used.

- 3 different volumetric limit size boxes are created:

1. Car Box: Limits the size of mesh around Ahmed Body up to $15 \mathrm{~mm}$.

2. Wake Box: Limits the size of mesh around wake region up to $10 \mathrm{~mm}$.

3. Underbody Box: Limits the size of mesh under the body up to $10 \mathrm{~mm}$.

The mesh is composed of 2112223 tetrahedral elements. Figure 4 shows the generated mesh.

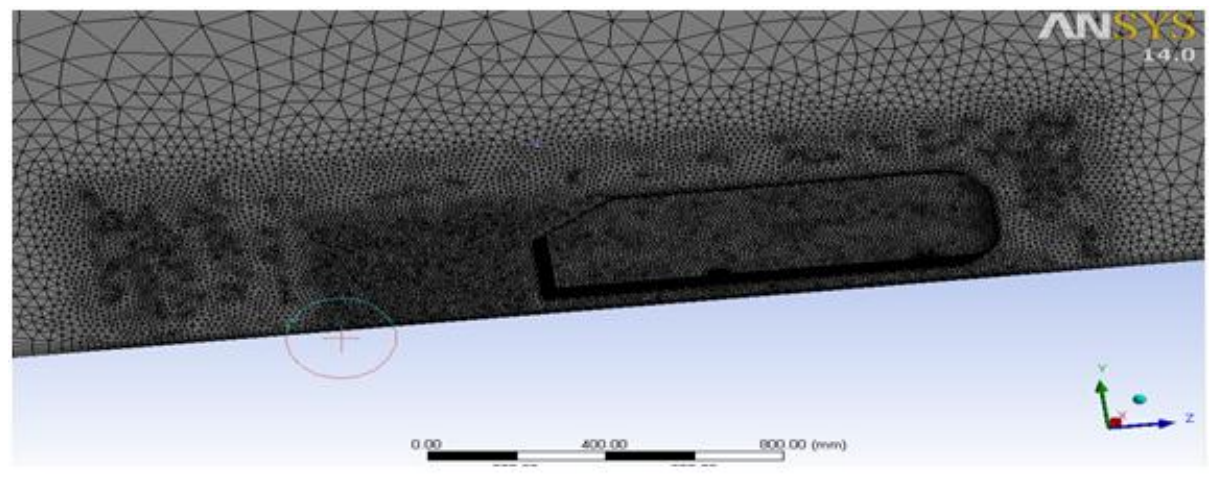

Figure 3: Mesh of Ahmed body

\subsection{Steady Flow Numerical Simulation:}

Computational resources for the steady flow numerical simulations are a Dell workstation (1 CPU 1.8 GHz and 8GB RAM) and Windows 8 OS.

Realizable k-epsilon model with non-equilibrium wall function for near wall treatment is used with Inlet velocity $\mathrm{V}=40 \mathrm{~m} / \mathrm{s}$ and turbulence intensity is about $1 \%$ and turbulence viscosity ratio is 10 at inlet, turbulence intensity is about $5 \%$ and turbulence viscosity ratio is 10 at outlet. Density of air is $1.225 \mathrm{~kg} / \mathrm{m}^{3}$, temperature is $288.16 \mathrm{~K}$ and viscosity is $1.7894 \mathrm{e}^{-05} \mathrm{~kg} / \mathrm{m}^{4}$. Boundary conditions are - uniform velocity at inlet, uniform pressure at outlet, symmetry at lateral and at top wall of the model and moving wall at floor.

Solution method utilizes pressure-velocity coupling scheme as coupled with gradient: least square cell based method, pressure as standard and setting the momentum, turbulence kinetic energy, turbulence dissipation rate as first order upwind for the initial 100 iterations and correspondingly as second order upwind for the next 500 iterations. Moreover, turbulence viscosity factor is taken as 0.8 for the initial 100 iterations and consequently taken as 0.95 for the remaining 500 iterations.

\section{Simulation Results}

The drag and lift coefficients are calculated for the 10 different manipulations of rear slant angle of the Ahmed body ranging from $0^{0-40^{\circ}}$. The minimum drag is obtained for the rear slant angle, $\varphi=7.5$ degree for the attached flow range of angles which exists up to $30^{\circ}$ and thereafter, the flow becomes unattached at rear slant and correspondingly the values of drag and lift tend to be randomly dispersed. 
Table 1: Steady flow numerical simulation results

\begin{tabular}{|l|l|l|}
\hline Rear slant angle $(\varphi)$ (In degrees) & $\mathbf{C}_{\mathbf{d}}$ & $\mathbf{C}_{\mathbf{l}}$ \\
\hline 0 & $2.50031 \mathrm{e}-01$ & $-1.17457 \mathrm{e}-01$ \\
\hline 5 & $2.37215 \mathrm{e}-01$ & $-1.49709 \mathrm{e}-02$ \\
\hline 7.5 & $2.34631 \mathrm{e}-01$ & $2.92149-02$ \\
\hline 10 & $2.36738 \mathrm{e}-01$ & $8.29710 \mathrm{e}-02$ \\
\hline 12.5 & $2.41644 \mathrm{e}-01$ & $1.32483 \mathrm{e}-01$ \\
\hline 15 & $2.46833 \mathrm{e}-01$ & $1.85001 \mathrm{e}-01$ \\
\hline 20 & $2.61934 \mathrm{e}-01$ & $2.83622 \mathrm{e}-01$ \\
\hline 30 & $2.97872 \mathrm{e}-01$ & $3.47783 \mathrm{e}-01$ \\
\hline 35 & $2.94980 \mathrm{e}-01$ & $2.05502 \mathrm{e}-01$ \\
\hline 40 & $2.50360 \mathrm{e}-01$ & $8.36791 \mathrm{e}-03$ \\
\hline
\end{tabular}

The table portrays our findings indicating that drag coefficient continually decreases from $0^{0}$ reaching a minimum at $7.5^{\circ}$ and then continually increases for the attached flow range up to $30^{\circ}$.

Moreover, the lift coefficient linearly increases as the rear slant angle is increased from $0^{0}$ up till $30^{\circ}$ transitioning from negative (also called 'downforce') to positive value of lift. The lift coefficient approaches zero value at $7.5^{0}$. ${ }^{[12]}$

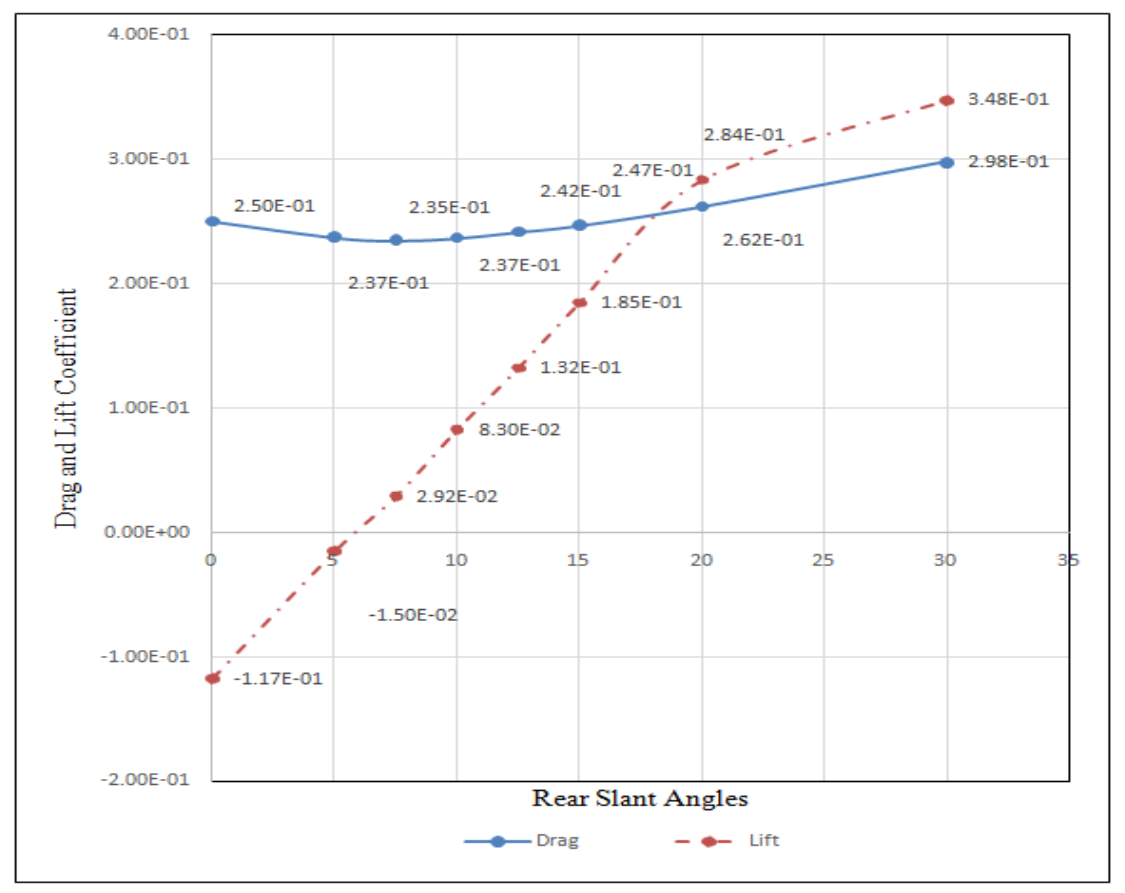

Figure 4: Graph between drag \& lift coefficients and different rear slant angles

The drag and lift coefficients are plotted on a graph (above). It is observed that the lift coefficient follows a linear increasing trend for rear slant angle ranging $0-20^{\circ}$. The minimum positive lift of 0.0292 is obtained for rear slant angle of $7.5^{\circ}$. Also, the drag coefficient decreases as rear slant is increased from $0^{0}$ up to $7.5^{\circ}$, where it reaches the minimum value of 0.2346 and then continually goes on increasing till $30^{\circ}$.

Beyond $30^{\circ}$, flow separation occurs and evidently drag and lift coefficient become randomly dispersed. 


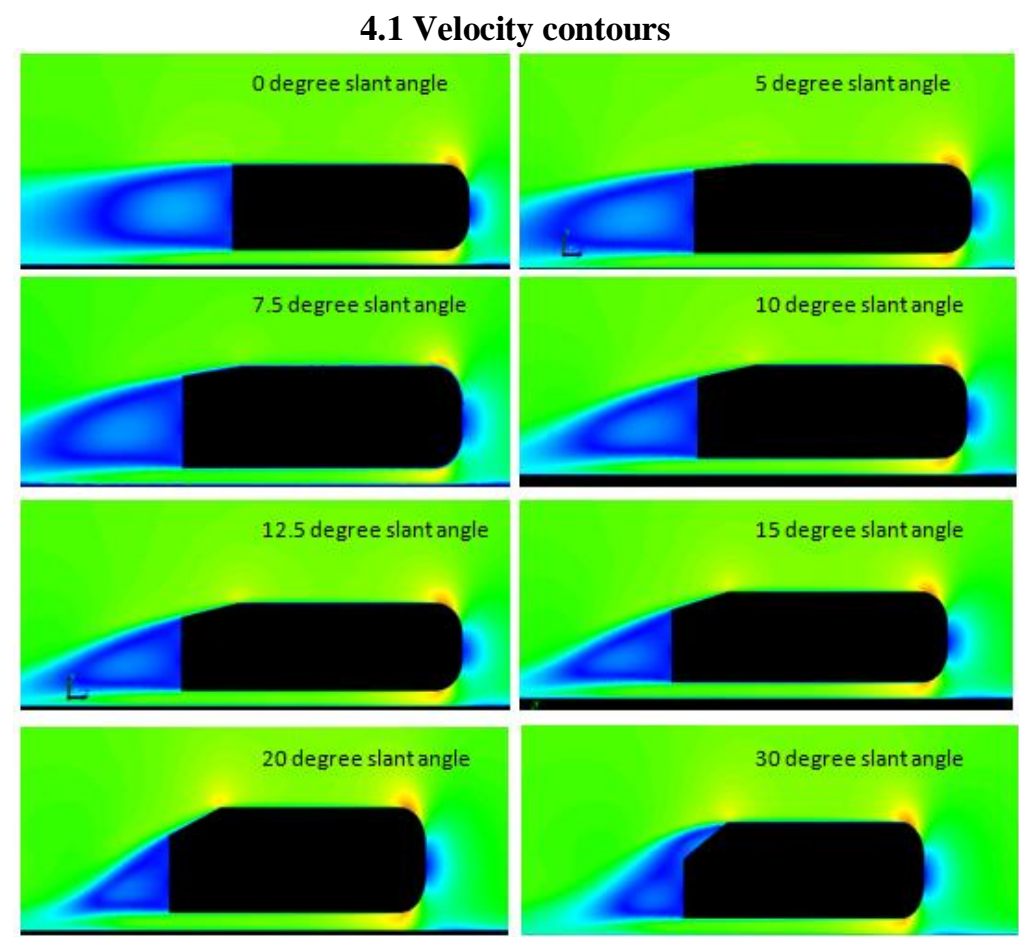

Figure 5: Velocity Contours at different rear slant angles

Gives us the velocity of air along different geometry sections of the Ahmed body. The green area corresponds to the velocity $40 \mathrm{~m} / \mathrm{sec}$, while blue area show low velocity and high pressure region and red area gives high velocity low pressure region.

The velocity contours clearly indicate that the wake region, and consequently the form drag, continually decreases as the rear slant angle is increased from $0-30^{\circ}$ and thereafter, boundary layer separation occurs on the rear slant. However, there is an increase in lift with increase in rear slant angle due to larger pressure difference generated between top and bottom surfaces of the vehicle, and this results in larger liftinduced drag. So, a trade-off between these two drag mechanisms is done to determine the optimum angle giving minimum drag coefficient.

As the wake area is reduced, the drag-causing eddies (vortices), generated due to the pressure difference between the top and bottom of the Ahmed body at the rear trailing end, come closer together. This results in less kinetic energy being dissipated due to these smaller eddies and consequently less compensation power required by the engine to propel the vehicle forward. It results in higher pressure being applied at the rear surface of the body and consequently less form drag. 


\subsection{Pressure distribution}
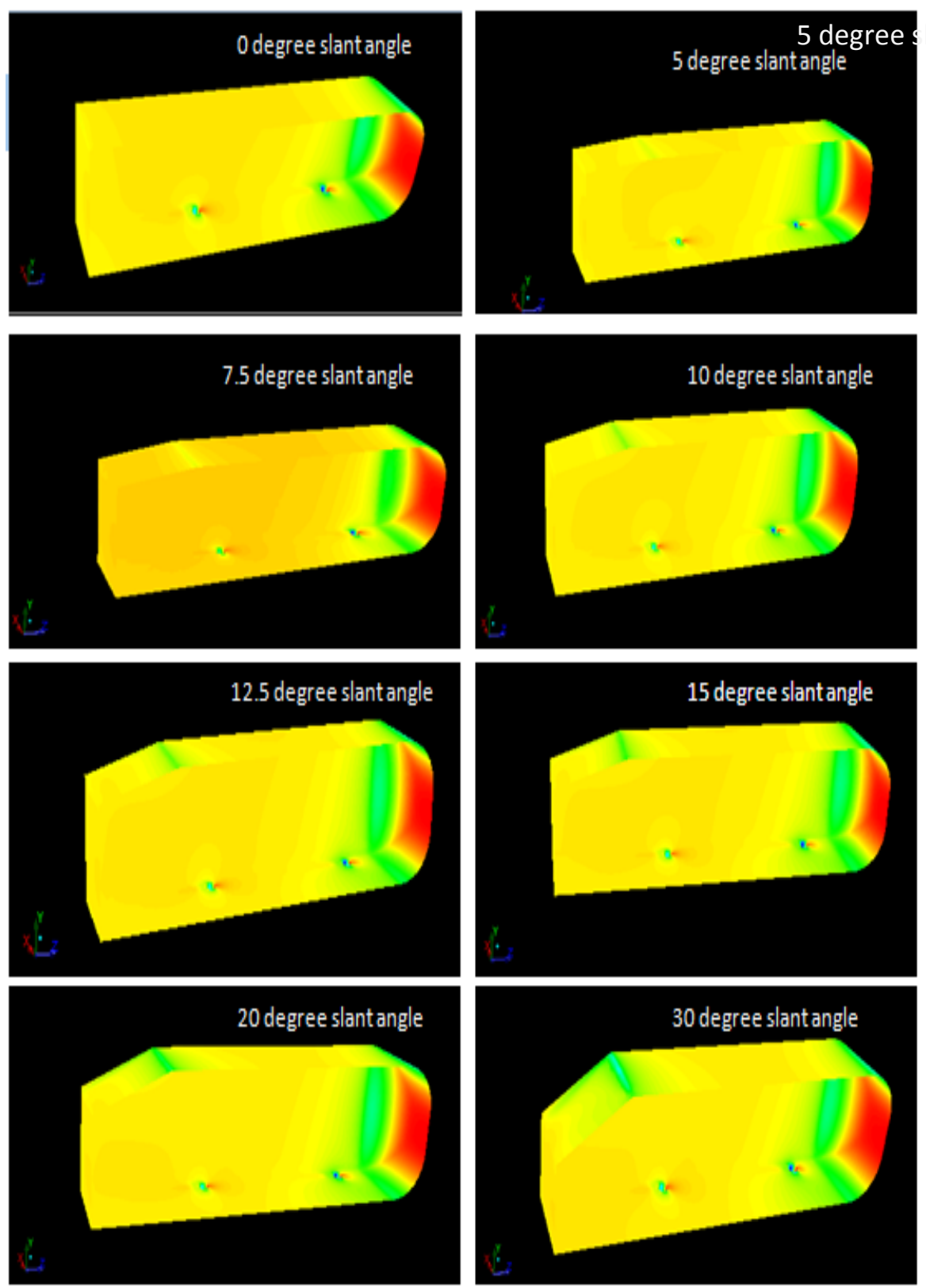

Figure 6: Pressure Distribution at different rear slant angles

The air becomes almost stagnant as it strikes the vehicle which results in air exerting very high pressure on front engine grill of the vehicle represented by the red area. The airflow then gets divided between the upper and lower surface of the vehicle. The higher pressure air on front surface accelerates as it travels over the curved nose surface of Ahmed body, causing the pressure to drop. This lower pressure creates lifts over the roof surface as the air passes over it. As the air continues to flow and make its way to the rear, a notch is created by the rear slant owing to flow separation, leaving a vacuum or low pressure space which the air is not able to fill properly. The resulting lower pressure creates lift that then acts upon the surface area of the rear slant.

For the multifarious slant angles, it is evident that at the initial section of the rear slant, the pressure goes on increasing (evident by the decreasing green area) with decreasing value of rear slant angle from $30^{\circ}-0^{\circ}$. This is due to less dramatic change in the direction of airflow, resulting in decreased formation of notch (low pressure or vacuum region). This results in more equalized pressure between the top and bottom surfaces of the Ahmed body and hence, reduced tendency of lift. 


\section{Conclusion}

CFD results for multifarious manipulations of the rear slant angle of the Ahmed Body are presented after the geometries are designed and then developed. Comparison and examination by plotting a graph of the lift and drag coefficients obtained for the numerous manipulations is done to determine the optimum rear slant angle giving minimum drag coefficient. The following conclusions can be drawn from the results:

1. The minimum coefficient of drag $\left(\mathbf{C}_{\mathbf{d}}\right)$ of $\mathbf{0 . 2 3 4 6}$ is obtained with configuration of Ahmed Body with $\mathbf{7 . 5}$ degree rear slant angle.

2. The drag increases as the rear slant angle is reduced and increased from $7.5^{\circ}$ down to $0^{\circ}$ and up to $30^{\circ}$ respectively.

3. The minimum positive coefficient of lift $\left(\mathbf{C}_{\mathbf{l}}\right)$ of $\mathbf{0 . 0 2 9 2}$ is obtained with configuration of Ahmed Body with 7.5 degree rear slant angle.

4. The lift coefficient constantly goes on increasing with increasing rear slant angle, for the attached flow range and it follows an almost linear trend for the $0^{0}-20^{\circ}$ range of angles, becoming non-linear thereafter.

5. The drag and lift coefficient becomes randomly dispersed after the flow becomes unattached after increasing rear slant angle to $30^{\circ}$ and beyond.

\section{References}

[1]. 'Sustainable ground transportation - review of technologies, challenges and opportunities', Ramesh K. Agarwal, Department of Mechanical Engineering \& Materials Science, Washington University in St. Louis, MO 63130, USA.

[2]. 'Some Salient Features of the Time - Averaged Ground Vehicle Wake', (SAE-TP-840300) Ahmed, S.R., Ramm, G., Faitin, G., 1984. Society of Automotive Engineers, Inc., Warrendale, PA.

[3]. 'Experimental and Computational Investigation of Ahmed Body for Ground Vehicle Aerodynamics', I. Bayraktar, D. Landman, and O. Baysal, SAE Technical Paper 2001-01-2742, 2001, doi:10.4271/2001-01-2742.

[4]. 'Flow Separations Generated by a Simplified Geometry of an Automotive Vehicle', A.Spohn, P. Gillieron, IUTAM Symposium: Unsteady Separated Flows, April 8-12, 2002, Toulouse, France.

[5]. 'Numerical Study of the Generic Sports Utility Vehicle Design with a Drag Reduction Add-On Device',Shubham Singh, M. Zunaid, Naushad Ahmad Ansari, Shikha Bahirani, Sumit Dhall, Sandeep Kumar, Department of Mechanical Engineering, Delhi Technological University, Hindawi Publishing Corporation Journal of Computational Engineering Volume 2014, Article ID 785294.

[6]. 'A New Aerodynamic Approach to Advanced Automobile Basic Shapes', A. Morelli, SAE Technical Paper 2001-01-0491, 2001.

[7]. 'Efficient Use of Computational Fluid Dynamics for the Aerodynamic Development Process in the Automotive Industry', P. Drage, A. Grabiel and G. Lindbichler, AIAA 2008-6735.

[8]. 'Flow and turbulence structures in the wake of a simplified car model (Ahmed Model)', H. Lienhart, C. Soots and S. Becker, SAE Technical Paper 2003-01- 0656, 2003.

[9]. 'Optimization of bluff body for minimum Drag in ground proximity', T. Han, D. C. Hammond, C. J. Sagi, AIAA Journal, Vol. 30, No. 4 (1992), pp. 882-889, doi: 10.2514/3.11005.

[10]. 'Characterization of a square back Ahmed body near wake flow', A. Lahayea, A. Leroya \& A. Kourtaa, 21 'eme Congress Francis de M'ecanique.

[11]. 'Best practice guidelines for handling Automotive External Aerodynamics with FLUENT', Version 1.2 (Feb 9th 2005), Marco Lanfrit, Fluent Deutschland GmbH Birkenweg 14a 64295 Darmstadt/Germany.

[12]. 'Investigation of relationship between drag and lift coefficients for a generic car model', Ivan Dobrev, Fawaz Massouh, BULTRANS-2014, Sep 2014, Sozopol, Bulgaria. BULTRANS 2014 Proceedings, pp.171-174. <Hal-01082895>

[13]. 'Experimental investigation and numerical simulation of the flow around an automotive model: Ahmed body', Ivan Korkischko \& Julio Romano Meneghini, 19th International Congress of Mechanical Engineering, November 5-9, 2007, Brasília, DF.

[14]. 'Numerical modeling of airflow over the Ahmed body', Y. Liu, Alfred Moser Air and Climate Group, Swiss Federal Institute of Technology, ETH-Zentrum WET A1, CH-8092 Zurich, Switzerland.

[15]. 'Fundamental of Aerodynamics', $5^{\text {th }}$ edition, John D. Anderson, McGraw-Hill Book Co. 